\title{
FABRICATION AND CHARACTERIZATION OF PASSIVE SAMPLER USING POLYMERIC INCLUSION MEMBRANE (PIM) AS DIFFUSION LAYER FOR PHOSPHATE MEASUREMENT
}

\section{Barlah Rumhayati ${ }^{\star}$, Adam Wiryawan, Layta Dinira, and Sakhiyah Afifah}

Chemistry Department, Faculty of Science, Brawijaya University

J. Veteran, Ketawanggede, Malang City, East Java 65145, Indonesia

*Correspondence: Tel. (0856)45145612, emails: rumhayati_barlah@ub.ac.id;

\section{ABSTRACT}

The PIM-passive sampler is a passive sampler using PIM as a diffusion layer. This research aimed to optimize the concentration of PIM plasticizer and the concentration of phosphate ions in bulk solution to produce a PIM-passive sampler for phosphate measurement with high accuracy. In this study, a passive sampler was made of a $15 \mathrm{ml}$ glass bottle containing $0.2 \mathrm{M} \mathrm{H}_{2} \mathrm{SO}_{4}$ solution as the receiving phase and PIM as a diffusion membrane. PIM was prepared by mixing polyvinyl chloride (PVC) as the base membrane, Aliquat 336-Cl as a carrier, and 1-decanol as a plasticizer. Sampling was done by immersing the passive sampler in the bulk phase solution for three hours. The phosphate concentrations in bulk and receiving phases were determined regularly every 30 minutes using the visible spectrophotometric method at a wavelength of $690 \mathrm{~nm}$. The results showed that the optimum concentration of 1 -decanol for PIM was $15 \% \mathrm{w} / \mathrm{w}$. The higher the plasticiser concentration, the PIM was oily, thus preventing the analyte from contacting the extractant. The range of phosphate ion concentrations in the sample that the PIM-passive sampler could detect was $0.1-1.3 \mathrm{mg} \mathrm{P} / \mathrm{L}$. The calibration curve for phosphate measurement was $\mathrm{C}_{\mathrm{TWA}}=$ 1.0664. Cs -0.0088 with a correlation coefficient $\left(R^{2}\right)$ was 0.9998 . This shows that the PIMpassive sampler can be used for the measurement of phosphate ions with high accuracy.

Keywords: PIM, passive sampler, phosphate, 1-decanol, Aliquat 336-Cl.

\section{INTRODUCTION}

Phosphate is a key nutrient in the process of algae growth blooming that is known as eutrophication. Phosphate sources in aquatic systems are from natural and human activities, one of which is synthetic fertilizers in agricultural activities. Among phosphate species, dissolved reactive phosphates are phosphate species consumed directly by aquatic biota (phytoplankton) in their metabolism. For this reason, it is necessary to monitor the concentration of phosphate in the aquatic environment in the context of water resources management. Eutrophication can emerge when the concentration of dissolved phosphate is more than $0.1 \mathrm{mg} / \mathrm{l}$. For the management of aquatic environments, phosphate detection at a concentration lower than $0.1 \mathrm{mg} / \mathrm{L}$ is needed. However, low phosphate concentrations are often not detected by spectrophotometric analysis methods developed by Murphy and Riley. 
[1]. Various methods were developed to preconcentrate phosphate from samples, for example, the coprecipitation method using Mg [2], HPLC [3], and SPE (Solid Phase Extraction) using zirconia as adsorbent [4]. These preconcentration methods were offline sample pretreatment technique that requires a large sample volume. Flow system based methods (online) called Flow injection analysis (FIA) have been developed for phosphate preconcentration using an anion exchange column [5] and using Polymeric Inclusion Membrane (PIM) [6]. The online preconcentration method is faster and more efficient than the offline method. However, in these methods, water sampling is carried out by grab sampling means that water samples are collected at one location and at one point in time, so we can not predict the time average concentration of phosphate in the aquatic environment.

The passive sampler was developed to overcome the obstacles, including time constraints, costs, Physico-chemical changes of analytes, and concentrations of analytes small enough to be directly measured. By using the passive sampler, the analyte from the bulk phase will be accumulated into the receiving phase in the sampler through the membrane phase as a diffusion layer for a certain period. The concentration of analyte detected by the passive sampler (formulated as time-weighted average concentration, CTWA) results from the concentration of analyte accumulated in the passive sampler by considering the diffusion rate of the analyte through the diffusion layer.

The existing passive sampler for phosphate detection uses the Chemcatcher with the Empore ${ }^{\mathrm{TM}}$ Anion SR extraction disk as the receiving phase [7]. After completion of sampling, the phosphate adsorbed on the disk was eluted using $0.5 \mathrm{M} \mathrm{HCl}$. This procedure was able to elute up to $90 \%$ phosphate. DGT-based passive samplers have also been developed for in situ measurements of phosphates in water using various types of binding gels, including Cerium oxide [8] and zirconium oxide [9]. As with the chemcatcher, the phosphate accumulated in the DGT binding gel can be eluted with acid before measurement of its concentration. The selection of acid for elution determines the success of the chemcatcher and DGT passive sampler for phosphate measurement.

Other passive sampler devices have been explored for measurement chemicals in aquatic system. In this device, PIM was used as a diffusion layer. Passive samplers with PIM layer have been investigated to measure the concentration of ammonia [10], arsenate [11], and zinc [12]. Studies on the PIMpassive sampler for phosphate measurement have not been reported. However, transport and preconcentration phosphate combined with Flow Injection Analysis (FIA) has been reported [6]. In the research, PIM was prepared from PVC as base polymer and Aliquat-336 chloride as a carrier without plasticizer. Plasticizers can be added to the PIM to increase the membrane flexibility and softness, and the flux of targeted ion [13]. Therefore, in this following research, the PIM consisted of PVC, Aliquat 336 chloride, and 1-decanol as plasticizer was fabricated and used as a diffusive layer of the passive sampler for phosphate measurement. The 
performance of passive sampler with PIM produced at various concentration was investigated in this study. The sampling rate (Rs) was calculated to determine the optimum concentration of plasticizer for PIM. In addition, the accuracy of phosphate measurement using PIM-passive sampler was also studied.

\section{METHODS}

\section{Materials, Tools, and Instrumentations}

Chemicals used for preparing PIM were high molecular weight Polyvinyl Chloride (PVC) (Merck), 1-decanol (Merck), Aliquat 336-Cl (Merck), and Tetrahydrofuran (THF) (Merck). Phosphate solution stock was prepared by dissolving $\mathrm{KH}_{2} \mathrm{PO}_{4}$ (Sigma) in demineralized water. $\mathrm{H}_{2} \mathrm{SO}_{4}$ (98\%, Merck) was used to prepare the receiving phase. Ammonium molybdate $\left(\left(\mathrm{NH}_{4}\right)_{6} \mathrm{Mo}_{7} \mathrm{O}_{24} .4 \mathrm{H}_{2} \mathrm{O}\right)$ (Sigma) and $\mathrm{SnCl}_{2} .2 \mathrm{H}_{2} \mathrm{O}$ (Merck) were reagents for phosphate detection. Concentrations of phosphate in bulk and receiving phases were determined using the Shimadzu UV 1601 Spectrophotometer.

\section{PIM Preparation}

PIM was prepared by dissolving the amount of PVC base polymer in $10 \mathrm{ml}$ of THF solvent. Afterwards, Aliquat $336-\mathrm{Cl}$ and 1decanol were added to obtain a total membrane weight of $300 \mathrm{mg}$. The carrier concentration was fixed at $30 \% \mathrm{w} / \mathrm{w}$ while the plasticizer concentration was varied from 5 to $25 \% \mathrm{w} / \mathrm{w}$.

The membrane solution was then poured into a glass mold with a diameter of 6 $\mathrm{cm}$ and covered with filter paper and a watch glass to evaporate the solvent slowly. After
24 hours, a thin, flexible and transparent membrane was formed. The membrane is a cut circle with a diameter of $2.2 \mathrm{~cm}$ and functions as a diffusion layer for the passive sampler.

\section{Passive sampler device}

The passive sampler device consists of a glass bottle with a volume of $15 \mathrm{~mL}$ with two top and bottom openings with a diameter of $1.6 \mathrm{~cm}$ and $2.2 \mathrm{~cm}$, respectively. The top opening is closed with a screw cap and is used to take the internal phase in the passive sampler bottle. The bottom opening is fitted with a screw cap with a hole with a diameter of $1.1 \mathrm{~cm}$. PIM was cut in a circle with a diameter of $1.1 \mathrm{~cm}$, clamped between the Teflon ring and rubber ring, and placed on the bottom opening. Parts of the passive sampler can be seen in Figure 1.

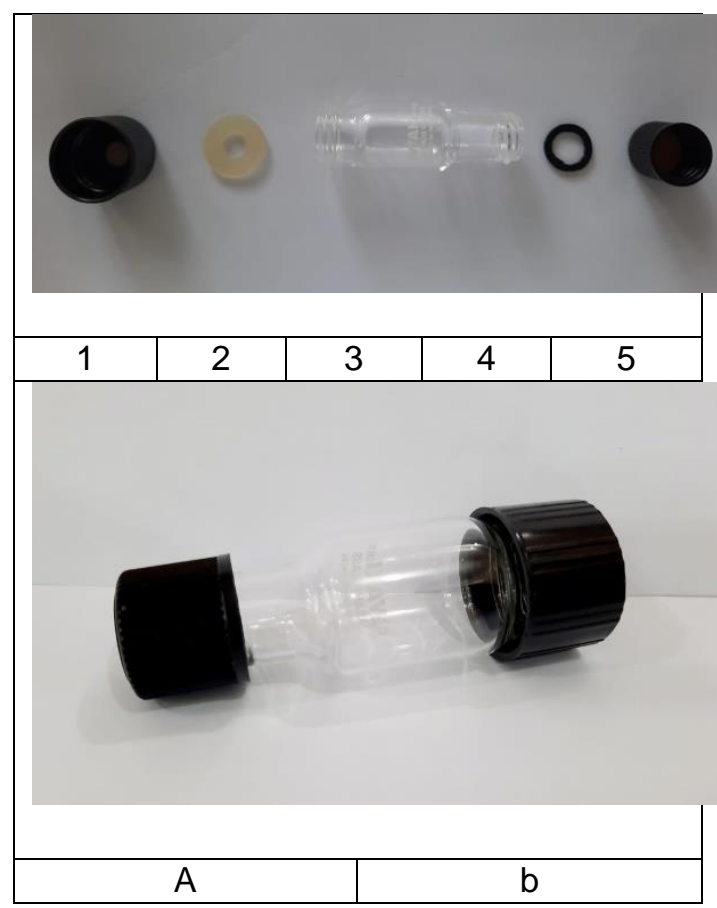

Figure 1. Parts of the passive sampler: (1) bottom cap, (2) Teflon ring, (3) passive sampler bottle, (4) rubber ring, (5) top cap; and ready-to-use passive sampler: (a) the top part, (b) the bottom part. 


\section{Passive Sampling Experiment}

A passive sampling experiment was conducted in the laboratory. The parts of the passive sampler were assembled by incorporating Aliquat 336 chloride-decanol based PIM and $15 \mathrm{~mL}$ of $0.2 \mathrm{M} \mathrm{H}_{2} \mathrm{SO}_{4}$ as the receiving phase. The passive samplers were placed in a polypropylene test tube rack in a vertical position where the PIM side was facing down. The test tube rack and the passive samplers were positioned inside a container containing $10 \mathrm{~L}$ of $0.1-2.0 \mathrm{mg} \mathrm{P} / \mathrm{L}$ phosphate solution as the bulk phase.

The aquarium pump was attached to one side of the container to imitate water flow as occurs in a natural aquatic environment.

Sampling was carried out for 3 hours. In every 30 minute, both the bulk and receiving phase were sampled for $4 \mathrm{~mL}$ and the phosphate concentration was determined.

Phosphate concentrations measured using a passive sampler (- $\mathrm{C}_{\text {TWA }}$ ) can be calculated using Eq. 1 below [14].

$$
\mathrm{C}_{\mathrm{TWA}}=\frac{\mathrm{Cr} \cdot \mathrm{Vr}}{\text { Rs.t }}
$$

$\mathrm{C}_{r}$ is the phosphate concentration in the receiving phase $(\mathrm{mg} / \mathrm{L}) . V_{r}$ is the volume of the receiving phase (L). $R_{s}$ is the sampling rate ( $\mathrm{L} / \mathrm{min}$ ) obtained from the slope of the linear curve between the equivalent volume $\left(V_{\text {eq }}\right)$ to the sampling time ( $t$, minutes), as shown in Eq. 2.

$$
\begin{aligned}
& V_{e q}=R_{s} \cdot t \\
& V_{\text {eq }}=\mathrm{m}_{\mathrm{r}} / \mathrm{C}_{\mathrm{s}},
\end{aligned}
$$

$V_{\text {eq }}$ can be calculated by Eq. 3, where $\mathrm{m}_{\mathrm{r}}$ is the mass of the phosphate ion in the receiving phase $(\mathrm{mg})$ and $\mathrm{Cs}$ is the concentration of phosphate in the bulk phase or sample (mg / L).

\section{Determination of phosphate concentration}

The concentration of phosphate ion was determined using standard procedure as stated in Standard Methods [15]. For determination, $10 \mathrm{ml}$ of sample was reacted with $0.4 \mathrm{ml}$ of molybdate reagent and $0.05 \mathrm{ml}$ of $\mathrm{SnCl}_{2}$ reagent, then shaken well and waited for 10-12 minutes. The absorbance of the solution was determined using UV-Vis spectrophotometre at wavelength of $690 \mathrm{~nm}$.

\section{RESULTS AND DISCUSSION}

\section{Characteristics of PIM}

PIM is the development of a liquid membrane after a bulk liquid membrane (BLM), an emulsion liquid membrane (ELM), and a supported liquid membrane (SLM). PIM has the same way of working as SLM, which is capable of simultaneous extraction and reverse extraction. Low carrier stability as an extractant in SLM [16], [17] can be overcome by using PIM because the release of the carrier can be prevented [13]. PIM is made by using a thermoplastic base polymer, for example, PVC. The polymer chains are straight, so there are no cross-links between the polymer chains, causing PVC to dissolve easily in solvents such as THF. The mechanical strength of PIM comes from the intermolecular forces and the entanglement between the chains [18].

Aliquat 336-chloride (trialkylmethylammonium chloride) is often used as a carrier for PIM. The three alkyl groups in Aliquat 336$\mathrm{Cl}$ are mixtures of the $\mathrm{C}_{8}-\mathrm{C}_{10}$ chain [19]. PIM with a composition of $70 \%$ PVC and $30 \%$ 
Aliquat 336-chloride (\% w / w) has been used as a diffusion membrane in simultaneous phosphate measurements using flow analysis. [6]. The composition used in the study adopted PIM, which is used for the extraction of arsenate ions [20].

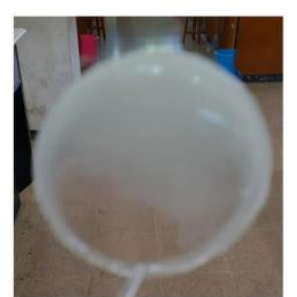

PIM 2A

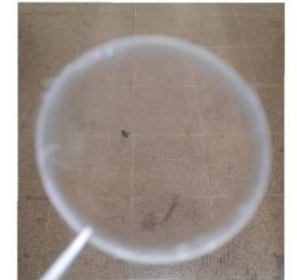

PIM 2B

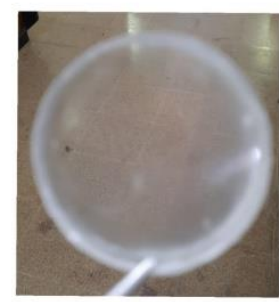

PIM 2C

Figure 2. PIMs were prepared using PVC, Aliquat 336-Chloride, and 1-decanol at a composition of $55 \%: 30 \%: 15 \%(\mathrm{w} / \mathrm{w})$ from three batches $(A, B$, dan $C)$.

As shown in Figure 2, the PIM produced is physically transparent and thin. The presence of parts that appear cloudy on the PIM is due to differences in the humidity of the mold used from three batch at the same PIM composition. To prevent this, the mold must be completely dry before use. Passive sampler test, the transparent part of the membrane was cut in a circle with a diameter of $2.2 \mathrm{~cm}$. By using a glass mold with a diameter of $6 \mathrm{~cm}$, the resulting membrane has a thickness of $30 \pm 0.5 \mu \mathrm{m}$. The resulting membrane did not undergo swelling during the sampling process ( 3 hours) where the weight of the membrane before and after sampling was relatively the same, namely $0.057 \pm 0.009$ grams.

\section{Optimization of Plasticizer Concentration}

The intermolecular forces contained in PIM are mainly Van der Waals forces which are weak and non-specific. On the other hand, the stronger polar-polar interactions only occur in polar functional groups, namely the $\mathrm{C}-\mathrm{Cl}$ groups in PVC. This can cause the membrane to become stiff, inflexible and reduce the diffusion of analytes across the membrane [21].

A plasticizer is added to increase the flexibility of the membrane so that the analyte can diffuse better. The polar group of plasticizer interact with the polar groups in the membrane so that the membrane becomes flexible. In addition, plasticizer molecules increase the distance between polymer molecules, thereby reducing the strength of intermolecular forces [21]. The concentration of plasticizer in PIM affects the transport and accumulation of phosphate ions in the passive sampler, so that it affects the accuracy of the passive sampler as a device for the measurement of phosphate ions. To determine the membrane composition that produces good transport and measurement of phosphate ions, in this research, 1-decanol was used as a plasticizer at various concentrations even though the extractant Aliquat 336-Cl contains 2$5 \%(w / w)$ 1-decanol [19].

The effect of plasticiser concentration on the accumulation of phosphate ions in the passive sampler is shown in Table 1. It can be seen in Table 1 that PIM made of PVC/Aliquat 336-Chloride/1-decanol was able to accumulate phosphate in the receiving phase. The amount of accumulated phosphate increased with an increase in the 1-decanol concentration up to $20 \%(w / w)$. 
When using 25\% plasticiser, the amount of phosphate accumulates was less.

Based on phosphate concentration in the receiving phase, the volume equivalent (Veq) can be calculated using Eq. 3, while using Eq. 4, the phosphate sampling rate $\left(R_{s}\right)$ can be determined. Based on the sampling rates obtained (Table 2), the C TwA of phosphate could be determined from Eq. 1 . Based on Figure 3, it can be seen that PIM with a composition of 55:30:15 (\% w/w) can be used as a diffusion layer of a passive sampler for phosphate measurement with high accuracy (98.8\%).
The low concentration of plasticiser causes the membrane to become stiff and easily damaged. Using PVC as the base polymer, the theoretical minimum concentration of 1 -decanol is $18 \% \mathrm{w} / \mathrm{w}$. Gibbons and Kusy [22] indicates that membrane performance will improve when using a plasticiser near the theoretical minimum concentration. From the research results, 1 -decanol with a concentration of $15 \%$ $(w / w)$ is the optimum concentration for PIM to be used as a diffusive layer in a passive sampler for phosphate measurement.

Table 1. The measured concentration of phosphate in the receiving phase of a passive sampler with PIM diffusive layer at various concentration of 1-decanol as a plasticizer

\begin{tabular}{cccccc}
\hline $\begin{array}{c}\text { Time (minutes) } \\
\text { / Plasticizer } \\
\begin{array}{c}\text { concentration } \\
(\% \mathrm{w} / \mathrm{w})\end{array}\end{array}$ & \multicolumn{5}{c}{ Measured phosphate concentration in the receiving phase $(\mathrm{C}, \mathrm{mg} / \mathrm{L})$} \\
\cline { 2 - 6 } & 5 & 10 & 15 & 20 & 25 \\
\hline 0 & 0.000 & 0.000 & 0.000 & 0.000 & 0.000 \\
30 & $0.286 \pm 0.037$ & $0.347 \pm 0.123$ & $0.434 \pm 0.123$ & $0.260 \pm 0.123$ & $0.347 \pm 0.123$ \\
60 & $0.477 \pm 0.061$ & $0.607 \pm 0.123$ & $0.694 \pm 0.123$ & $0.694 \pm 0.123$ & $0.521 \pm 0.123$ \\
90 & $0.733 \pm 0.061$ & $0.781 \pm 0.123$ & $0.954 \pm 0.123$ & $0.954 \pm 0.123$ & $0.868 \pm 0.000$ \\
120 & $0.998 \pm 0.061$ & $1.215 \pm 0.123$ & $1.215 \pm 0.123$ & $1.215 \pm 0.123$ & $1.128 \pm 0.123$ \\
150 & $1.258 \pm 0.061$ & $1.388 \pm 0.123$ & $1.388 \pm 0.123$ & $1.475 \pm 0.123$ & $1.301 \pm 0.123$ \\
180 & $1.432 \pm 0.061$ & $1.562 \pm 0.123$ & $1.648 \pm 0.123$ & $1.648 \pm 0.123$ & $1.388 \pm 0.123$ \\
\hline
\end{tabular}

Table 2. The sampling rate (Rs) of phosphate measured with PIM-passive sampler

\begin{tabular}{cc}
\hline PVC : Aliquat 336-Cl : 1-decanol & Rs (L/minutes) \\
$(\% \mathrm{w} / \mathrm{w})$ & 0.00008 \\
$65: 30: 5$ & 0.00008 \\
$60: 30: 10$ & 0.00008 \\
$55: 30: 15$ & 0.00010 \\
$50: 30: 20$ & 0.00008 \\
$45: 30: 25$ & \\
\hline
\end{tabular}




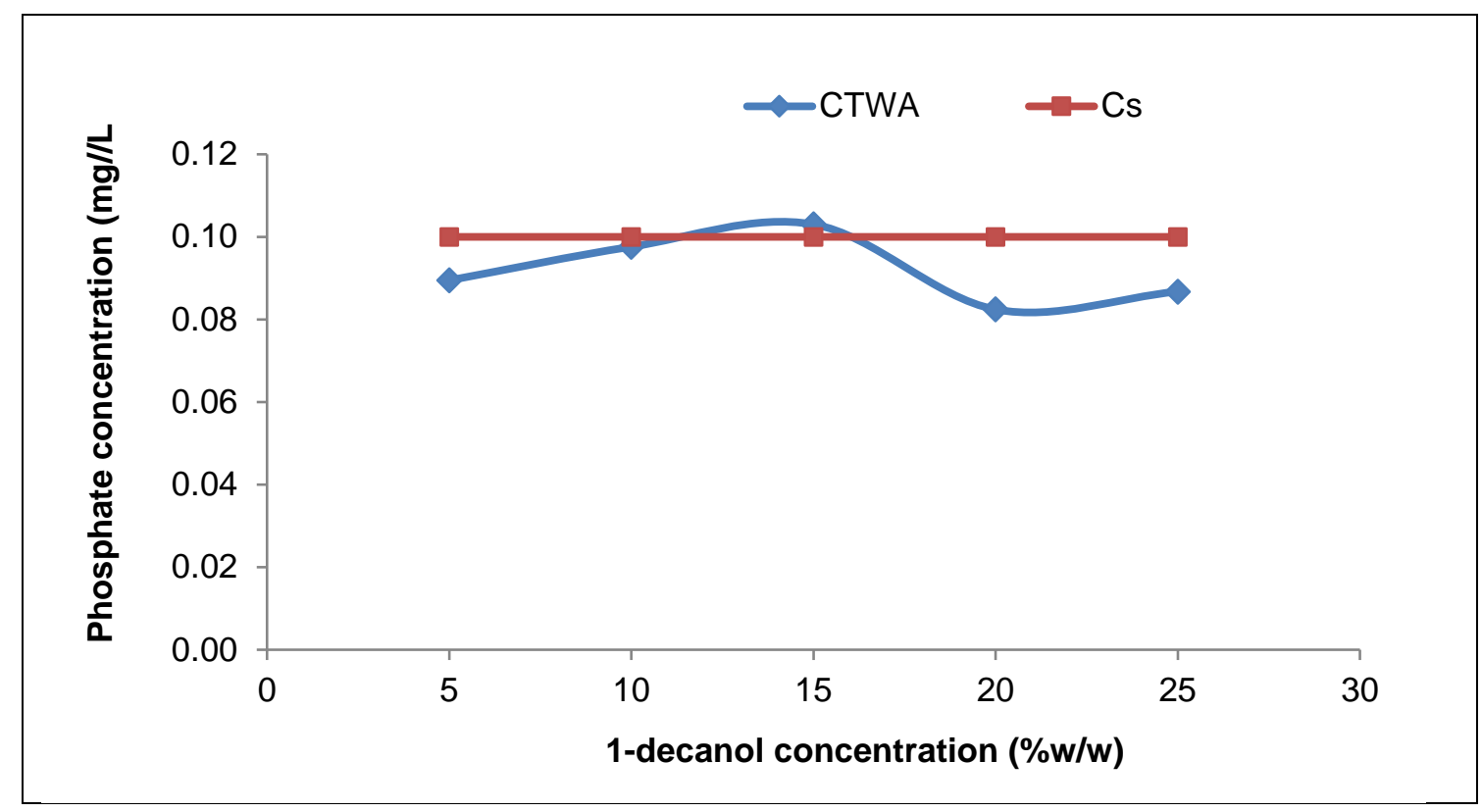

Figure 3. The effect of 1-decanol concentration on the $\mathrm{C}_{\mathrm{TWA}}$ (blue) and $\mathrm{C}_{\mathrm{s}}(\mathrm{red})$ of phosphate.

Transport of phosphate through PIM in the passive sampler is determined by the carrier used for the PIM. In this study, Aliquat 336-chloride $\left(\mathrm{R}_{4} \mathrm{~N}^{+} \mathrm{Cl}-\right)$ was used as a carrier. Aliquat 336-chloride (n-octhylmethyl ammonium chloride, TOMAC) reacts as an anion-exchanger by forming an-ion pair with anion from the aqueous phase. By using sulphate ion in the receiving phase, the phosphate transport could be proposed as below:

a. Bulk phase

$2 \mathrm{H}_{2} \mathrm{PO}_{4}^{-}{ }_{(\mathrm{aq})}^{-}+2\left(\mathrm{R}_{4} \mathrm{~N}^{+} \mathrm{Cl}^{-}\right)(\mathrm{m})$

$\rightleftharpoons 2 \mathrm{Cl}_{(\mathrm{aq})}^{-}+2\left(\mathrm{R}_{4} \mathrm{~N}^{+} \mathrm{H}_{2} \mathrm{PO}_{4}^{-}\right)(\mathrm{m})$

b. Receiving phase

$2\left(\mathrm{R}_{4} \mathrm{~N}^{+} \mathrm{H}_{2} \mathrm{PO}_{4}{ }^{-}\right)(\mathrm{m})+\mathrm{SO}_{4}{ }^{2-}($ aq)

$\rightleftharpoons \mathrm{q} 2 \mathrm{H}_{2} \mathrm{PO}_{4}{ }^{-}(\mathrm{aq})+\left(\mathrm{R}_{4} \mathrm{~N}^{+}\right)_{2} \mathrm{SO}_{4}{ }^{2-}(\mathrm{Fm})$

\section{Calibration of Passive Sampler for Phosphate Measurement}

Calibration of passive sampler is needed prior to the field sampling using a passive sampler for determining the phosphate concentration at certain period of time. The passive sampler was used to measure phosphate at concentration range of $0.1-1.3 \mathrm{mg} \mathrm{P} / \mathrm{L}$. These concentration range of phosphate is primarily found in natural aquatic environments.

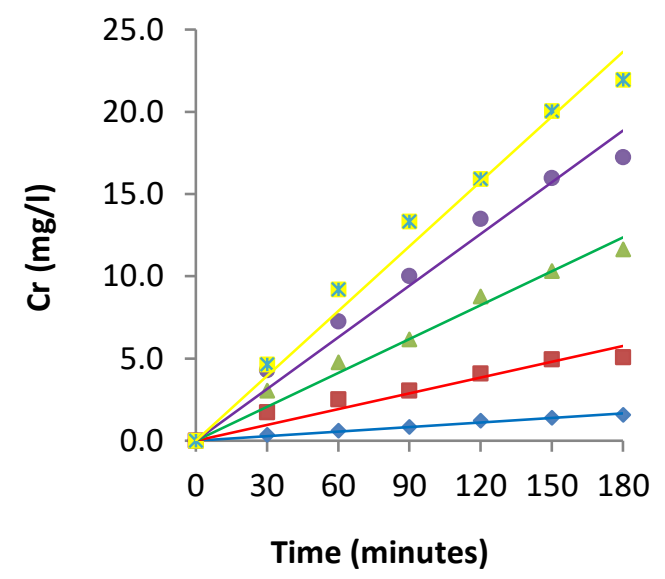

Figure 4. Phosphate concentration in the receiving phase of PIM-passive sampler for the following bulk phase concentrations: 0.1 (blue), 0.3 (red), 0.7 (green), 1.0 (purple), and $1.3 \mathrm{mg}$ $\mathrm{P} / \mathrm{l}$ (yellow). Passive sampler used PIM, which consisted of $15 \% \mathrm{w} / \mathrm{w}$ of 1-decanol. 
Figure 4 shows linear diffusion of phosphate from the bulk phase to the receiving phase solution at five concentrations. It can be suggested that the gradient concentration of phosphate and sulphate between in bulk and receiving solutions causing the increase of the sampling rate of the phosphate at high concentration.

.A linear calibration curve for phosphate measurement using a PIMpassive sampler was obtained at phosphate concentration in the bulk solution up to 1.3 $\mathrm{mg} / \mathrm{L}$, as shown in Figure 5 with the equation $C_{\text {TWA }}=1.0664 . C_{s}-0.0088\left(R^{2}=0.9998\right)$ at 3 hours sampling period. Based on this equation, the concentration of phosphate in the field (Cs) during sampling period time can be calculated from the $\mathrm{C}_{\text {TWA }}$.

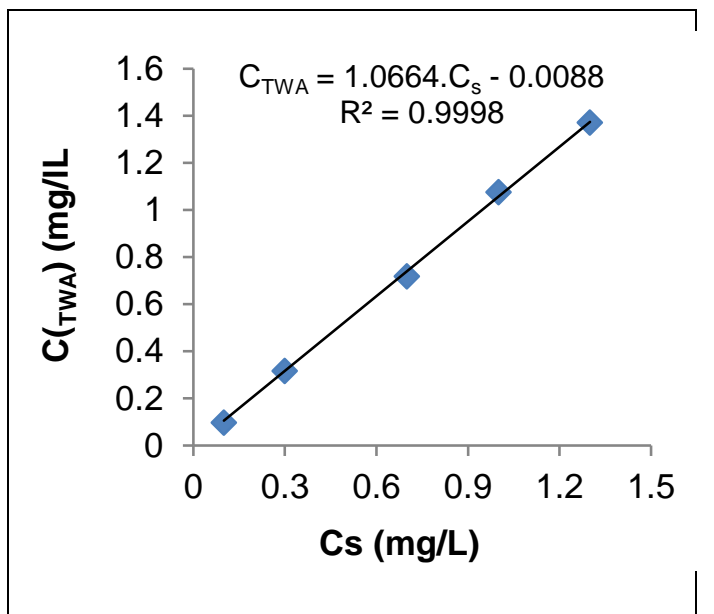

Figure 5. Calibration curve for phosphate measurement using PIM-passive sampler.

\section{CONCLUSION}

A PIM-based passive sampler could be used for phosphate measurement in an aquatic system. The optimum composition of PIM was 55\% PVC, 30\% Aliquat 336 chloride, and 15\% 1-decanol (\%w/w). PIM could be used as a diffusion layer in the passive sampler device to measure phosphate concentration up to $1.3 \mathrm{mg} / \mathrm{l}$ with up to $99 \%$ accuracy. For applications in natural aquatic environments, the presence of other anions in the sample may affect the detection of phosphate ions, so that it needs to be observed in further research.

\section{ACKNOWLEDGMENTS}

The authors would like to thank Universitas Brawijaya for providing research funding assistance in the 2020 Professor and Doctoral Research Grant scheme.

\section{REFERENCES}

[1] J. Murphy \& J. P. Riley, "A modified single solution method for the determination of phosphate in natural waters," Anal.Chim.Acta, vol. 27, pp. 31-36, 1962,

doi: 10.1016/S0003-2670(00)88444-5.

[2] P. Rimmelin \& T. Moutin, "Reexamination of the MAGIC method to determine low orthophosphate concentration in seawater," Anal.Chim.Acta, vol. 548, no. 1-2, pp. 174-182, 2005,

doi: 10.1016/j.aca.2005.05.071.

[3] J. L. Haberer \& J. A. Brandes, "A high sensitivity, low volume HPLC method to determine soluble reactive phosphate in freshwater and saltwater," Mar Chem, vol. 82, no. 3-4, pp. 185-196, 2003,

doi: 10.1016/S0304-4203(03)00069-0.

[4] M. Okumura, K. Fujinaga, Y. Seike, \& K. Hayashi, "A Simple in situ Preconcentration Method for Phosphate Phosphorus in Environmental Waters by Column Solid Phase Extraction Using Activated Carbon Loaded with Zirconium," Anal Sci, vol. 14, no. 2, pp. 417-419, 1998, doi: 10.2116/analsci.14.417. 
[5] Y. Narusawa, "Flow-injection spectrophotometric determination of silicate, phosphate and arsenate with online column separation," Anal.Chim.Acta, vol. 204, pp. 53-62, 1988, doi: 10.1016/S0003-2670(00)86345-X.

[6] E. A. Nagul, C. Fontaz, I. D. McKelvie, R. W. Cattrall, \& S. D. Kolev, "The use of a polymer inclusion membrane for separation and preconcentration oforthophosphate in flow analysis," Anal.Chim.Acta, vol. 83, pp. 82-90, 2013,

doi: 10.1016/j.aca.2013.07.052.

[7] J. Knutsson, S. Rauch, \& G. M. Morrison, "Performance of a passive sampler for the determination of time averaged concentrations of nitare and phosphate in water," Env. Sci Process. Impacts, vol. 15, p. 933, 2013, doi: 10.1039/c3em00038a.

[8] F. Tan, Y. Wang, Y. Wang, S. Ren, Y. Cui, \& D. $\mathrm{Xu}$, "Ceria oxide nanoparticle-based diffusive gradients in thin films for in situ measurement of dissolved reactive phosphorus in waters and sewage sludge," Env. Sci Pollut Res Int, vol. 27, no. 10, pp. 11138-11146, 2020, doi: 10.1007/s11356-019-07220-5.

[9] Q. Sun, Y. Chen, D. Xu, Y. Wang, \& S. Ding, "Investigation of potential interferences on the measurement of dissolved reactive phosphate using zirconium oxide-based DGT technique," J Env. Sci China, vol. 25, no. 8, pp. 1592-1600, 2013, doi: 10.1016/s1001-0742(12)60140-5.

[10] M. I. G. S. Almeida, A. M. L. Silva, R. A. Coleman, V. J. Pettigrove, R. W. Cattrall, \& S. D. Kolev, "Development of a passive sampler based on a polymer inclusion membrane for total ammonia monitoring in freshwaters," Anal Bioanal Chem, vol. 408, no. 12, pp. 3213-3222, 2016, doi: 10.1007/s00216-016-9394-2.

[11] R. Vera, E. Antico, \& C. Fontas, "The use of a polymer inclusion membrane for arsenate determination in groundwater," Water, vol. 10, p. 1093, 2018, doi: 10.3390/w10081093.

[12] M. I. G. S. Almeida, C. Chan, V. J. Pettigrove, \& R. W. Cattrall, "Development of a passive sampler for Zinc(II) in urban pond waters using a polymer inclusion membrane," Env. Poll, vol. 193, pp. 233-239, 2014, doi: 10.1016/j.envpol.2014.06.040.

[13] L. D. Nghiem, P. Mornane, I. D. Potter, J. M. Perera, R. W. Cattrall, \& S. D. Kolev, "Extraction and transport of metal ions and small organic compounds using polymer inclusion membranes (PIMs)," J Membr Scie, vol. 281, pp. 7-41, 2006, doi: 10.1016/j.memsci.2006.03.035.

[14] L. Ahrens, A. Daneshvar, Anna. E. Lau, \& J. Kreuger, "Characterization of five passive sampling devices for monitoring of pesticides in water," $J$ Chrom A, vol. 1405, pp. 1-11, 2015, doi: 10.1016/j.chroma.2015.05.044.

[15] R. B. Baird, A. D. Eaton, \& E. W. Rice, Standard Methods for the examination water and wastewater, 23rd ed. USA: American Public Health Association, American Water Works Association, Water Environment Federation, 2017. ISBN: 9780875532875

[16] J. de Gyves \& E. R. de San Miguel, "Metal ion separations by supported liquid membranes," Ind Eng Chem Res, vol. 38, no. 6, pp. 2182-2202, 1999, doi: 10.1021/ie980374p.

[17] A. M. Sastre, A. Kumar, J. P. Sukla, \& R. K. Singh, "Improved techniques in liquid membrane separations: an overview," Sep Purif Meth, vol. 27, no. 2, pp. 213-298, 1998, doi: $10.1080 / 03602549809351641$.

[18] R. P. Wool, "Polymer entanglements," Macromolecules, vol. 26, no. 7, pp. 1564-1569, 1993, doi: 10.1021/ma00059a012. 
[19] A. Stojanovic, M. Lämmerhofer, D. Kogelnig, S. Schiesel, M. Sturm, M. Galanski, \& W. Lindner, "Analysis of quartenary ammonium and phosphonium ionic liquids by reversed-phase high-performance liquid chromatography with charged aerosol detection and unified calibration," JChromatogr A, vol. 1209, pp. 179-187, 2008,

doi: 10.1016/j.chroma.2008.09.017.

[20] R. Vera, A. Enriqueta, \& C. Fontaz, "The used of polymer inclusion membrane for arsenate determination in groundwater," Water, vol. 10, pp. 1093-1102, 2018,

doi: 10.3390/w10081093.
[21] J. K. Sears \& J. R. Darby, J.K. Sears, J.R. Darby, Technology of Plasticizers, , New York, 1982, p. 1174. New York: John Wiley \& Sons, 1982.

doi: 10.1002/pol.1982.130200810

[22] W. S. Gibbons \& R. P. Kusy, "Influence of plasticizer configurational changes on the dielectric characteristics of highly plasticized poly(vinyl chloride)," Polymer, vol. 39, no. 14, pp. 31673178, 1998,

doi: 10.1016/S0032-3861(97)10001-5. 